\title{
A Rare Case of Metastasis to the Thyroid Gland from Renal Clear Cell Carcinoma 11 Years after Nephrectomy and Concurrent Primary Esophageal Carcinoma
}

\author{
Mohammad Saud Khan $\left(\mathbb{D},{ }^{1}\right.$ Veena Balakrishnan Iyer, ${ }^{2,3}$ and Neha Varshney ${ }^{4}$ \\ ${ }^{1}$ Department of Internal Medicine, University of Toledo Medical Center, Toledo, OH 43614, USA \\ ${ }^{2}$ Department of Hematology and Oncology, Rhode Island Hospital, Providence, RI, USA \\ ${ }^{3}$ Department of Oncology, University of Toledo Medical Center, Toledo, OH 43614, USA \\ ${ }^{4}$ Department of Pathology, University of Toledo Medical Center, Toledo, OH 43614, USA \\ Correspondence should be addressed to Mohammad Saud Khan; mohammad.khan2@utoledo.edu
}

Received 10 December 2017; Accepted 28 January 2018; Published 2 April 2018

Academic Editor: Katsuhiro Tanaka

Copyright (C) 2018 Mohammad Saud Khan et al. This is an open access article distributed under the Creative Commons Attribution License, which permits unrestricted use, distribution, and reproduction in any medium, provided the original work is properly cited.

Renal cell carcinoma is known to cause metastasis to unusual sites, which can be both synchronous or metachronous. Thyroid gland is a rare site for metastasis, but when it occurs, renal cell carcinoma is the most common primary neoplasm. We report the case of a 81-year-old female patient who had a significant medical history of right clear cell renal carcinoma with adrenal metastasis. She underwent right radical nephrectomy and adrenalectomy followed by radiofrequency ablation of left adrenal metastasis and systemic chemotherapy with sunitinib. Eleven years later, she presented with dysphagia and was found to have distal esophageal adenocarcinoma. On imaging, there was incidental detection of a left renal mass lesion and a right thyroid nodule, which on histopathology and immunohistochemistry were confirmed to be clear cell carcinoma of renal origin.

\section{Introduction}

Thyroid gland is a rare site for clinically detectable metastasis despite having rich blood supply [1]. Renal cell carcinoma (RCC) is the most common primary neoplasm that metastasizes to thyroid gland and accounts for more than $50 \%$ of clinically recognized cases [2]. Other neoplasm frequently identified with metastasis to thyroid gland includes breast, lung, skin, and colon cancers. Metastasis accounts for 2-3\% of all thyroid malignancies detected clinically $[3,4]$. However, the incidence of thyroid metastasis has been reported to be higher (ranging from 1.9 to $22.4 \%$ ) on autopsy studies [5]. We report the case of a 81-yearold female patient who developed recurrence of RCC with thyroid metastasis after 11 years following nephrectomy of initial tumor.

\section{Case Report}

A eighty-one-year-old African American female patient presented to the hospital with the complaints of dysphagia and loss of appetite for past few weeks. She had a past medical history of right RCC, clear cell carcinoma subtype with bilateral adrenal metastasis (T3b, N0, and M1) diagnosed in May 2006. At that time, she was treated with right radical nephrectomy and right adrenalectomy followed by radiofrequency ablation of left adrenal metastasis and systemic chemotherapy with sunitinib. She tolerated the treatment well with adequate control of her malignant disease for 11 years. Recently, in June 2017, she presented with the complaints of dysphagia predominantly for solid food, loss of appetite, and generalized fatigability. Physical examination was unremarkable. Esophagogastroduodenoscopy with endoscopic ultrasound showed a distal esophagus mass causing high-grade stricture extending up to the adventitia with no mediastinal lymphadenopathy. Metallic esophageal stent was placed in, and biopsies were obtained which showed adenocarcinoma of esophagus (T3NOM0). Computed tomography (CT) of the chest and abdomen showed distal esophageal thickening along with incidental findings of solid mass lesion involving the inferior pole of the 


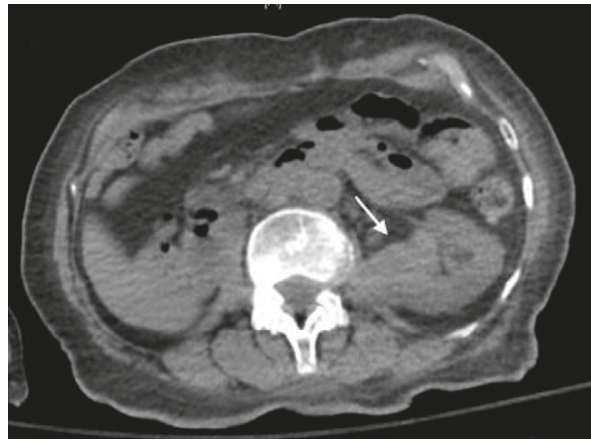

(a)

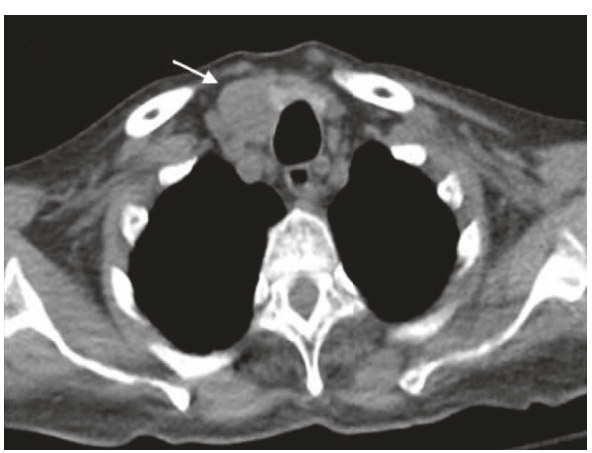

(b)

Figure 1: (a) Axial CT scan of the abdomen showing a mass lesion involving the inferior pole of the left kidney (arrow), breaching the renal capsule and infiltrating into adjacent retroperitoneal space. (b) Axial CT scan of the chest at the level of thyroid showing a well-defined hypodense nodule in the right lobe of the thyroid gland (arrow).

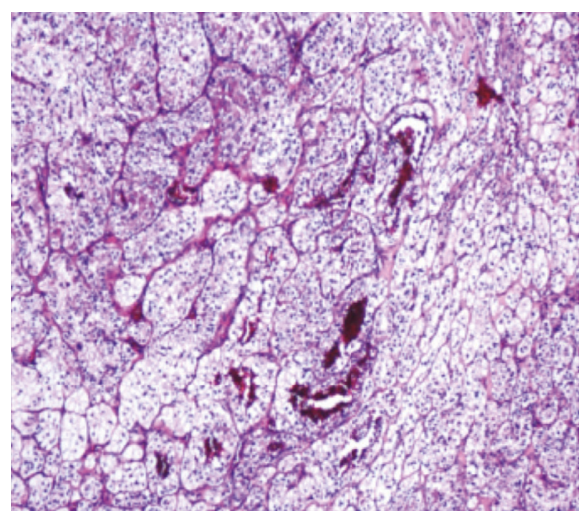

(a)

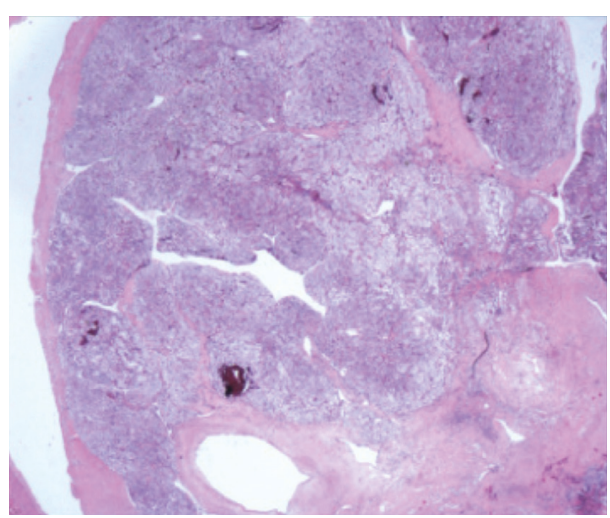

(b)

Figure 2: (a) Clear cells with increased vasculature consistent clear cell carcinoma of the kidney (40x, H\&E). (b) Clear cell carcinoma invading renal vein $(10 x, H \& E)$.

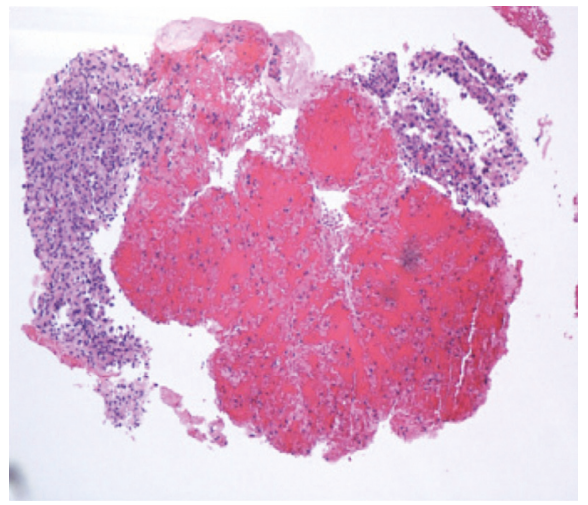

(a)

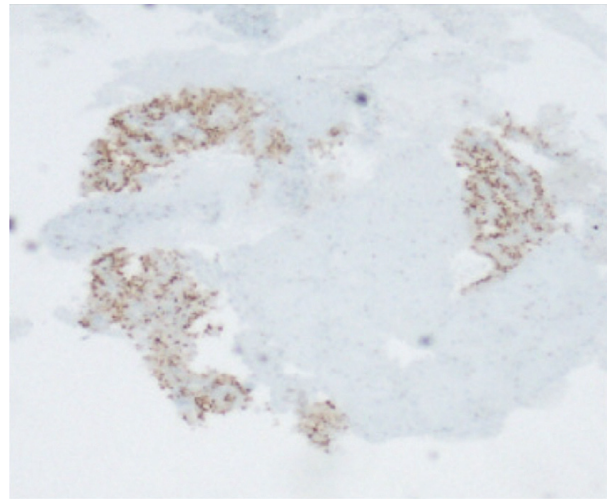

(b)

FIGURE 3: (a) Cell block (4x, H\&E) of the thyroid showing atypical cells, which are PAX-8 positive (b) consistent with metastasis of renal origin.

left kidney measuring $3.4 \times 2.6 \mathrm{~cm}$ in the largest dimension (Figure 1(a)) and a well-defined hypodense nodule in the right lobe of the thyroid gland measuring $2.1 \times 2.8 \mathrm{~cm}$ in the largest dimension (Figure 1(b)). Biopsy of renal mass was done which showed neoplastic cells with clear cytoplasm arranged in nests and mitotic figures suggesting clear cell carcinoma (Figure 2). Fine-needle aspiration (FNA) from the thyroid nodule also identified neoplastic clear cells on cytology raising possibility of metastasis from RCC (Figure 3(a)). This was confirmed with immunohistochemical stain, which showed atypical cells to be positive for PAX8 (Figure 3(b)) and CAIX while negative for TTF-1. Positron emission 
tomography (PET) scans showed increased uptake in the distal esophagus, left renal mass, and right thyroid nodule. A diagnosis of concurrent distal esophageal adenocarcinoma along with left renal RCC recurrence and thyroid metastasis was made. The patient was planned for left nephrectomy and thyroidectomy. However, the patient wished to opt out for any surgical intervention or aggressive medical therapy and preferred to be treated with comfort care measures. The patient was treated with palliative intention and died 2 months later.

\section{Discussion}

RCC accounts for approximately 3-4\% of all adult malignancies [6]. It is the most common renal malignancy and the second most common malignancy of urological tract $[6,7]$. RCC is more common in males compared to females (ratio of $2: 1$ ) and occurs predominantly in the 6th to 8th decade of life with a median age of 64 years [6]. Major histopathological subtypes include clear cell carcinoma, papillary carcinoma, chromophobe carcinoma, collecting duct carcinoma, medullary carcinoma, and unclassified categories [8]. Clear cell carcinoma is the most common subtype making up to $75 \%$ of cases of RCC [9]. RCC is known to metastasize in unpredictable manner. The metastasis may be detected at the time of diagnosis (synchronous) or may be found years after the diagnosis and treatment (metachronous) [7]. The most common route of metastasis is hematogenous and likely involves lung, liver, bone, lymph nodes, adrenal gland, and brain. Head and neck metastasis are less frequent and of which thyroid is the most commonly involved site. Late recurrences and distant metastasis ranging from few months to several years after initial diagnosis are a notable feature of RCC. It has been estimated that $20-30 \%$ of patients including those who have undergone nephrectomy with curative intent will develop recurrence and out of these $50 \%$ will relapse distantly $[7,10]$. Most of the recurrences are within 3 years of surgery, but delayed recurrences even after decades have been reported [11]. The longer the recurrence-free time from surgery, the more likelihood is of a true cure.

In majority of cases, thyroid metastasis is metachronous with average time of development being 9.4 years following resection of primary RCC [12]. But cases of RCC recurring with thyroid metastasis have been reported as late as 26 years [1]. These metastatic thyroid lesions may pose diagnostic challenge since they often occur years after treatment of primary lesion. RCC metastasis to thyroid can be asymptomatic and may be detected incidentally or may present with symptoms of palpable neck swelling, thyroid enlargement, dysphagia, dysphonia, or dyspnea [3]. Although metastasis to the thyroid gland may be suspected in patients with history of RCC, it is difficult to make a definitive preoperative diagnosis. Metastatic thyroid lesions usually appear as solid, hypoechoic, well-demarcated nodules with irregular border and increased vascularity on ultrasound imaging [13] and cold nodules on radioisotope uptake studies. These radiological features are nonspecific, and it is not possible to distinguish between primary and secondary thyroid neoplasms on imaging. FNA cytology serves as a reliable tool for establishing preoperative diagnosis.
However, sometimes it is difficult to distinguish metastasis from tumors of thyroid, which can have clear cell component on FNA cytology alone. In these cases, immunohistochemistry is helpful and aids in differential diagnosis. Some of the traditional immunohistochemical markers for renal cell carcinoma are cytokeratin, vimentin, and CD10 [14]. However, recently novel markers for RCC have been identified which have increased sensitivity and specificity for identifying RCC. These include anti-carbonic anhydrase IX (CAIX), anti-human kidney injury molecule-1 (hKIM-1), and PAX8 [15]. The immunohistochemical markers used for identifying primary thyroid malignancies are thyroglobulin, thyroid transcription factor-1 (TTF-1), and calcitonin. In our case, immunohistochemical stains were positive for CAIX and PAX-8 and negative for TTF-1.

Definitive diagnosis of metastatic RCC is usually made by histopathological examination after thyroidectomy. Surgical resection with either partial or total thyroidectomy should be performed if thyroid gland is the only site for metastasis. Prognosis is good in this group $[3,16]$. Patients with disseminated disease have poor prognosis and should undergo thyroidectomy only for palliation for compressive symptoms [16].

\section{Conclusion}

A thyroid nodule in a patient with a history of renal malignancy should be considered as potentially metastatic. Clinical manifestation and radiographic findings are nonspecific and are unable to distinguish between primary and secondary thyroid neoplasms. FNA cytology and immunohistochemistry are helpful in establishing diagnosis and should be obtained in suspected cases.

\section{Conflicts of Interest}

The authors declare that no conflicts of interest exist regarding the publication of this paper.

\section{References}

[1] M. K. Nakhjavani, H. Gharib, J. R. Goellner, and J. A. van Heerden, "Metastasis to the thyroid gland. A report of 43 cases," Cancer, vol. 79, no. 3, pp. 574-578, 1997.

[2] H. Chen, T. L. Nicol, and R. Udelsman, "Clinically significant, isolated metastatic disease to the thyroid gland," World Journal of Surgery, vol. 23, no. 2, pp. 177-180, 1999.

[3] A. Y. Chung, T. B. Tran, K. T. Brumund, R. A. Weisman, and M. Bouvet, "Metastases to the thyroid: a review of the literature from the last decade," Thyroid, vol. 22, no. 3, pp. 258-268, 2012.

[4] L. Hegerova, M. L. Griebeler, J. P. Reynolds, M. R. Henry, and H. Gharib, "Metastasis to the thyroid gland: report of a large series from the Mayo Clinic," American Journal of Clinical Oncology, vol. 38, no. 4, pp. 338-342, 2015.

[5] R. A. Willis, "Metastatic tumours in the thyroid gland," American Journal of Pathology, vol. 7, no. 3, pp. 187-208, 1931.

[6] R. L. Siegel, K. D. Miller, and A. Jemal, "Cancer statistics, 2016," CA: A Cancer Journal for Clinicians, vol. 66, no. 1, pp. 7-30, 2016.

[7] R. J. Motzer, N. H. Bander, and D. M. Nanus, "Renal-cell carcinoma," New England Journal of Medicine, vol. 335, no. 12, pp. 865-875, 1996. 
[8] G. Kovacs, M. Akhtar, B. J. Beckwith et al., "The Heidelberg classification of renal cell tumours," Journal of Pathology, vol. 183, no. 2, pp. 131-133, 1997.

[9] J. J. Hsieh, M. P. Purdue, S. Signoretti et al., "Renal cell carcinoma," Nature Reviews Disease Primers, vol. 3, p. 17009, 2017.

[10] R. C. Flanigan, S. C. Campbell, J. I. Clark, and M. M. Picken, "Metastatic renal cell carcinoma," Current Treatment Options in Oncology, vol. 4, no. 5, pp. 385-390, 2003.

[11] S. E. Eggener, O. Yossepowitch, J. A. Pettus, M. E. Snyder, R. J. Motzer, and P. Russo, "Renal cell carcinoma recurrence after nephrectomy for localized disease: predicting survival from time of recurrence," Journal of Clinical Oncology, vol. 24, no. 19, pp. 3101-3106, 2006.

[12] C. S. Heffess, B. M. Wenig, and L. D. Thompson, "Metastatic renal cell carcinoma to the thyroid gland: a clinicopathologic study of 36 cases," Cancer, vol. 95, no. 9, pp. 1869-1878, 2002.

[13] K. Kobayashi, M. Hirokawa, T. Yabuta et al., "Metastatic carcinoma to the thyroid gland from renal cell carcinoma: role of ultrasonography in preoperative diagnosis," Thyroid Research, vol. 8, no. 1, p. 4, 2015.

[14] L. D. Truong and S. S. Shen, "Immunohistochemical diagnosis of renal neoplasms," Archives of Pathology \& Laboratory Medicine, vol. 135, no. 1, pp. 92-109, 2011.

[15] A. R. Sangoi, M. Fujiwara, R. B. West et al., "Immunohistochemical distinction of primary adrenal cortical lesions from metastatic clear cell renal cell carcinoma: a study of 248 cases," American Journal of Surgical Pathology, vol. 35, no. 5, pp. 678-686, 2011.

[16] R. De Stefano, R. Carluccio, E. Zanni et al., "Management of thyroid nodules as secondary involvement of renal cell carcinoma: case report and literature review," Anticancer Research, vol. 29, no. 2, pp. 473-476, 2009. 


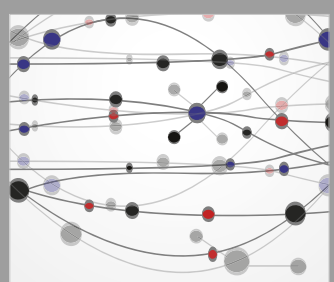

The Scientific World Journal
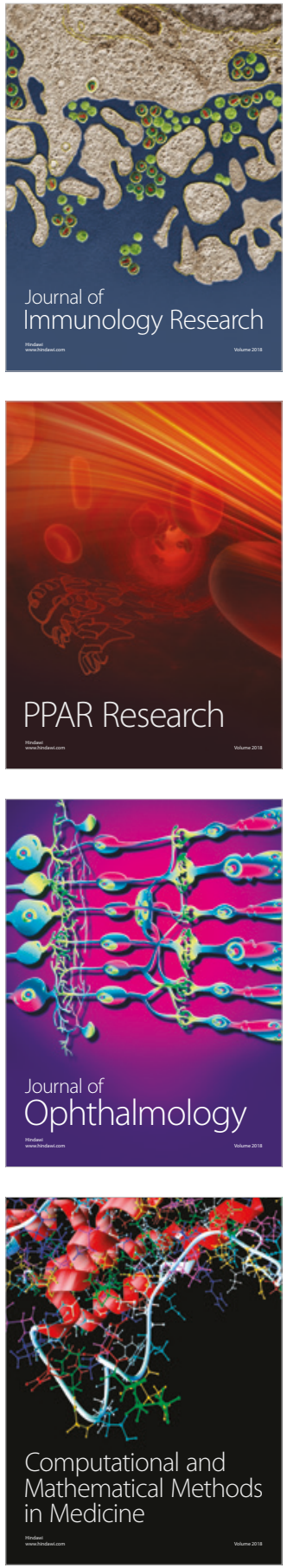

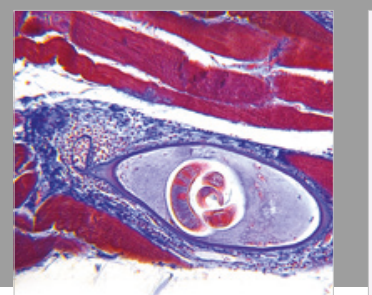

Gastroenterology Research and Practice

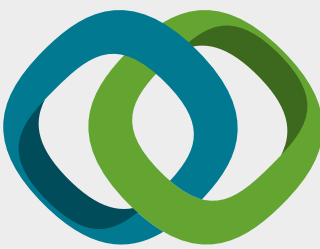

\section{Hindawi}

Submit your manuscripts at

www.hindawi.com
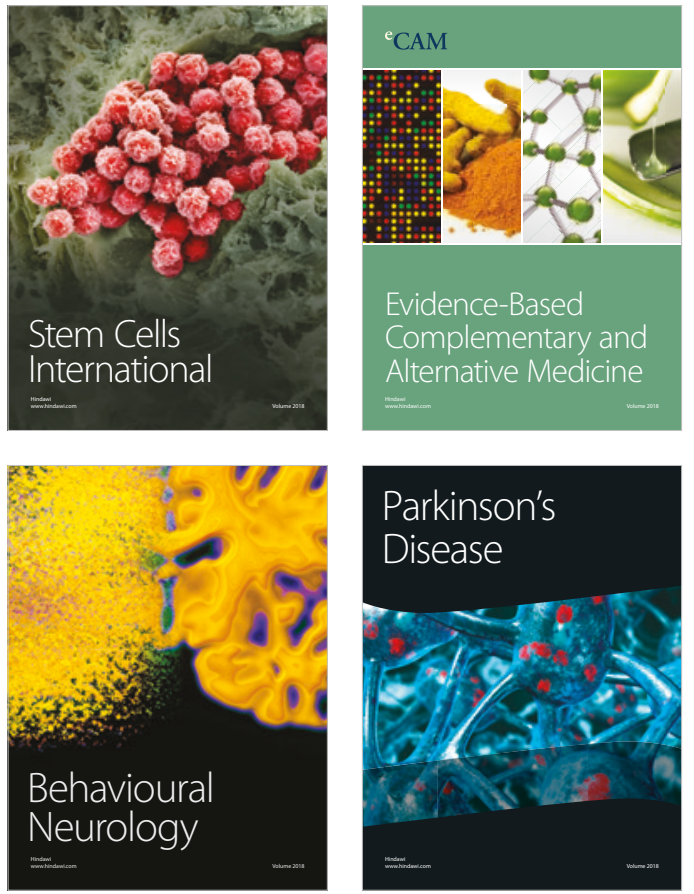

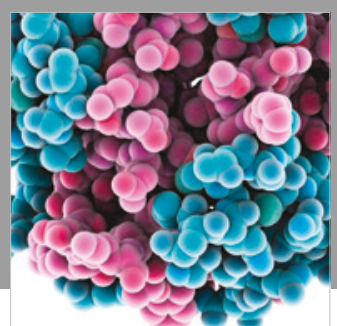

ournal of

Diabetes Research

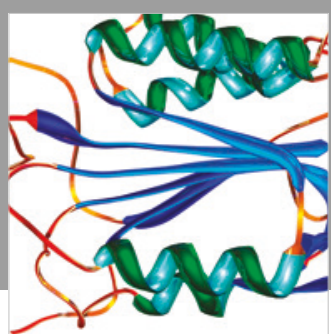

Disease Markers
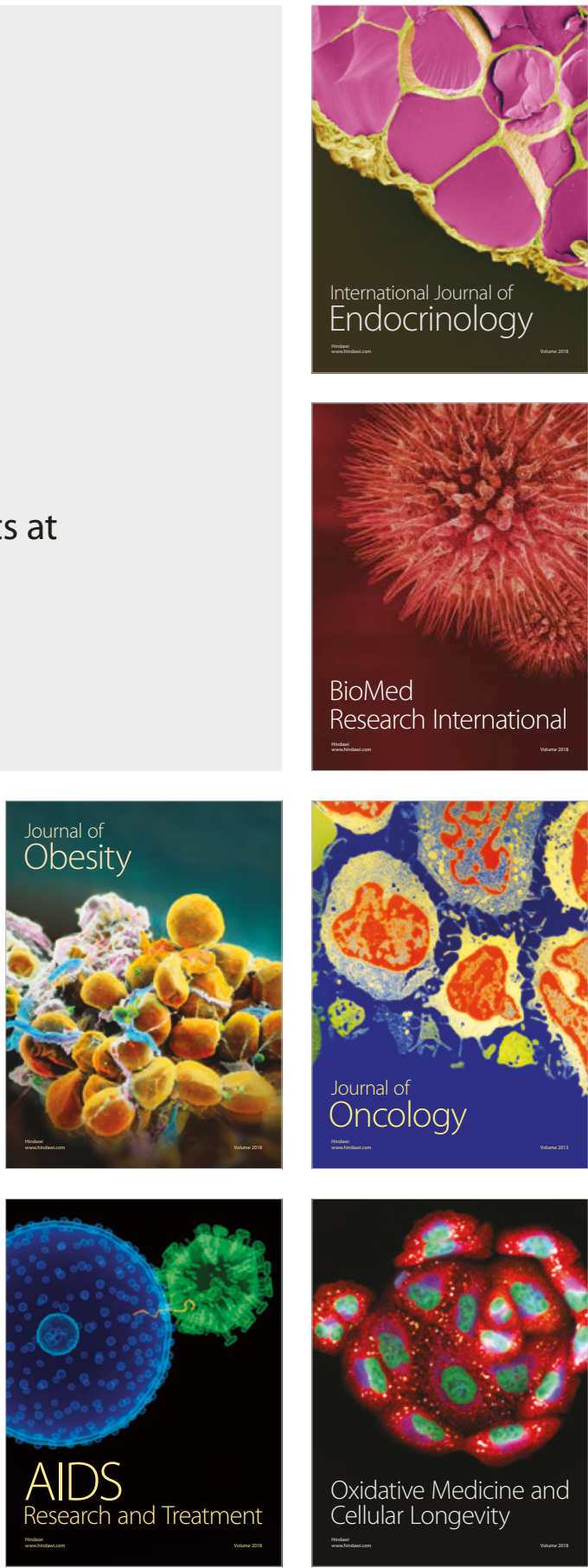